PROCEEDINGS OF THE

AMERICAN MATHEMATICAL SOCIETY

Volume 139, Number 6, June 2011, Pages 2009-2015

S 0002-9939(2010)10791-8

Article electronically published on December 1, 2010

\title{
THE STRUCTURE OF THE BOIJ-SÖDERBERG POSETS
}

\author{
DAVID COOK II
}

(Communicated by Irena Peeva)

\begin{abstract}
Boij and Söderberg made a pair of conjectures, which were subsequently proven by Eisenbud and Schreyer and then extended by Boij and Söderberg, concerning the structure of Betti diagrams of graded modules. In the theory, a particular family of posets and their associated order complexes play an integral role. We explore the structure of this family. In particular, we show that the posets are bounded complete lattices and the order complexes are vertex-decomposable, hence Cohen-Macaulay and squarefree glicci.
\end{abstract}

Boij and Söderberg recently conjectured in [4 a complete characterisation, up to multiplication by a positive rational, of the structure of Betti diagrams of finitely generated graded modules. Their conjectures were proven for the Cohen-Macaulay case in [7. These were further extended to arbitrary graded modules in 3]. This characterisation centered around constructing the convex hull of a particular class of Betti diagrams and showing that it is equal to a geometric realisation of a particular simplicial complex-the order complex of a poset.

The family of posets described in [4. Definition 2.3], which we refer to as the BoijSöderberg posets, is the focus of this paper. In particular, we study the structure of the posets and their associated order complexes. While no immediate applications are presented here, we hope that our results will help shed light on the mysterious relation between the decomposition of Betti tables and the associated modules.

In Section 1 we recall the relevant combinatorial definitions and explicitly define the Boij-Söderberg posets (Definition 1.1). Following this, in Section 2 we determine some basic structural results for the posets. In particular, we show that the Boij-Söderberg posets are indeed bounded complete lattices (Proposition 2.1). In Section 3 we discuss the recursive structure of the posets by finding a recursive atom ordering (see 1]) for each of the posets (Theorem 3.1). This allows us to conclude that the order complexes are vertex-decomposable, Cohen-Macaulay, and squarefree glicci (Corollary 3.2).

\section{Preliminaries}

1.1. Combinatorics. A simplicial complex $\Delta$, on a finite set $V$, is a set of subsets of $V$ closed under inclusion; elements of $\Delta$ are called faces. The dimension of a face $\sigma$ is $\# \sigma-1$ and of a complex $\Delta$ is the maximum of the dimensions of its faces.

Received by the editors June 10, 2010.

2010 Mathematics Subject Classification. Primary 05E45, 06B23, 13C14.

Key words and phrases. Boij-Söderberg theory, lattice, order complex, vertex-decomposable.

Part of the work for this paper was done while the author was partially supported by the National Security Agency under grant number H98230-09-1-0032.

(C)2010 American Mathematical Society Reverts to public domain 28 years from publication 
A complex whose maximal faces, called facets, are equi-dimensional is called pure, and a complex with a unique maximal face is called a simplex.

Given two simplicial complexes $\Delta$ and $\Gamma$ with disjoint vertex sets, we define the join of $\Delta$ and $\Gamma$ to be the simplicial complex

$$
\Delta \star \Gamma:=\{\sigma \cup \tau \mid \sigma \in \Delta, \tau \in \Gamma\} .
$$

If $\Delta=v$ is a vertex, then $v \star \Gamma$ is said to be the cone of $\Gamma$ with apex $v$.

Let $\sigma$ be a face of $\Delta$; then the link and deletion of $\sigma$ from $\Delta$ are given by

$$
\operatorname{link}_{\Delta} \sigma:=\{\tau \in \Delta \mid \tau \cap \sigma=\emptyset, \tau \cup \sigma \in \Delta\} \text { and } \operatorname{del}_{\Delta} \sigma:=\{\tau \in \Delta \mid \sigma \not \tau\} .
$$

Following [10, Definition 2.1], a pure complex $\Delta$ is said to be vertex-decomposable if either $\Delta$ is a simplex or there exists a vertex $v \in \Delta$, called a shedding vertex, such that both $\operatorname{link}_{\Delta} v$ and $\operatorname{del}_{\Delta} v$ are vertex-decomposable. Checking if a particular simplicial complex is vertex-decomposable can be done using a computer program such as [8]; in particular, the package described in [5] provides the appropriate methods.

A poset $P$ is a set with a partial ordering, that is, a binary relation " $\leq$ " over the set which is reflexive, antisymmetric, and transitive. Given $v, u \in P$ with $v \leq u$, the interval $[v, u]$ is the sub-poset $\{w \in P \mid v \leq w \leq u\}$. A chain is a sequence $v_{0}<v_{1}<\cdots<v_{p}$ of elements of $P$; such a chain is said to have length $p$. The poset $P$ is called pure if every maximal chain has the same length. The poset $P$ is bounded if there exists a unique minimal element, $\hat{0}$, and a unique maximal element, $\hat{1}$, of $P$.

Let $S$ be a subset of $P$. The meet of $S$, if it exists, is the infimum of $S$ and is denoted $\vee S$. Similarly, the join of $S$, if it exists, is the supremum of $S$ and is denoted $\wedge S$. If every distinct pair of elements in $P$ has a meet and a join, then $P$ is called a lattice. If every subset of $P$ has a meet and a join, then $P$ is called a complete lattice.

Let $P$ be a finite poset. For elements $x, y \in P$, we say that $y$ covers $x$, denoted by $x \rightarrow y$, if $x<y$ and $x<z \leq y$ implies $y=z$. In this case, we also say $x$ is covered by $y$. An atom of a bounded poset is an element which covers $\hat{0}$, the unique minimal element of $P$.

In [1], Björner and Wachs define a bounded pure poset $P$ to admit a recursive atom ordering if either $P$ has maximal chains of length one or there is an ordering of the atoms, $a_{1}, \ldots, a_{t}$, of $P$ which satisfies the properties:

(1) for $1 \leq j \leq t,\left[a_{j}, \hat{1}\right]$ admits a recursive atom ordering on its atoms $b_{1}, \ldots, b_{s}$ with the property that there exists a $1 \leq k \leq s$ such that $b_{l} \rightarrow a_{i}$ for some $1 \leq i<j$ if and only if $l \leq k$, and

(2) for $1 \leq i<j \leq t$, if $a_{i}, a_{j}<y$, then there is a $k<j$ and $z \in P$ such that $a_{k}, a_{j} \rightarrow z \leq y$.

Associated to every finite poset $P$ is the order complex, denoted $\Delta(P)$, which is a simplicial complex with faces given by chains in $P$. The facets of $\Delta(P)$ are exactly the maximal chains of $P$; hence $P$ is pure if and only if $\Delta(P)$ is pure. Notice that the minimal non-faces of $\Delta(P)$ are exactly the pairs of incomparable elements of $P$, so $\Delta(P)$ is a flag complex.

1.2. Boij-Söderberg posets and order complexes. In [4, Boij and Söderberg made a pair of conjectures about the possible graded Betti numbers of graded modules up to multiplication by positive rational numbers; the conjectures were 

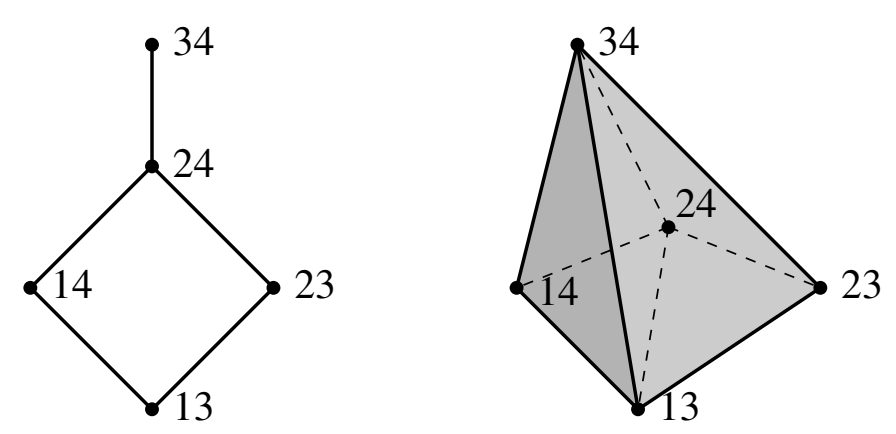

Figure 1. The Hasse diagram and the order complex of $\Pi_{(1,3),(3,4)}$

proven in [7] and [3]. In the course of their construction, they define a family of posets which we recall here.

Definition 1.1. Let $\underline{d}, \bar{d} \in \mathbb{Z}^{p+1}$ be strictly increasing sequences with $\underline{d}_{i} \leq \bar{d}_{i}$ for $0 \leq i \leq p$. Define the Boij-Söderberg poset of $\underline{d}$ and $\bar{d}$ to be the set $\Pi_{\underline{d}, \bar{d}}$ of strictly increasing sequences $d \in \mathbb{Z}^{p+1}$ such that $\underline{d}_{i} \leq d_{i} \leq \bar{d}_{i}$ for $0 \leq i \leq p$ endowed with the partial ordering defined by $d \leq e$ whenever $d_{i} \leq e_{i}$ for $0 \leq i \leq p$.

The Boij-Söderberg order complex of $\underline{d}$ and $\bar{d}$ is the order complex of the BoijSöderberg poset $\Pi_{\underline{d}, \bar{d}}$; that is, $\Delta\left(\Pi_{\underline{d}, \bar{d}}\right)$.

Notice that in 4 , the poset $\Pi_{\underline{d}, \bar{d}}$ has the associated pure diagrams as vertices, but these are equivalent as pure diagrams are in bijection to strictly increasing sequences in $\mathbb{Z}^{p+1}$. We further note that $\Delta\left(\Pi_{\underline{d}, \bar{d}}\right)$ is a pure complex by [4, Proposition 2.7].

Example 1.2. Let $\underline{d}=(1,3)$ and $\bar{d}=(3,4)$. Figure 1 gives both $\Pi_{d, \bar{d}}$ and its order complex. Note that we use concatenation of the sequence to label the vertices; e.g. we label $(2,4)$ as 24 .

Further, the family of Boij-Söderberg posets contains the family of posets of bounded root sequences described in [7, Section 8], where it is shown that the supernatural cohomology tables of root sequences in a bounded range give a geometric realisation of the order complex of the associated bounded root sequences. These are in turn used to prove the Boij-Söderberg conjectures.

\section{BAsic Structure}

Let $\underline{d}, \bar{d} \in \mathbb{Z}_{0}^{p+1}$ be strictly increasing sequences with $\underline{d}_{i} \leq \bar{d}_{i}$ for $0 \leq i \leq p$. First we see that the order complex has very nice structure.

Proposition 2.1. The poset $\Pi_{\underline{d}, \bar{d}}$ is a bounded complete lattice.

Proof. Let $D=\left\{d^{(1)}, \ldots, d^{(n)}\right\} \subset \Pi_{\underline{d}, \bar{d}}$. Define $l, u \in \mathbb{Z}^{p+1}$ by $l_{i}=\min _{j} d_{i}^{(j)}$ and $u_{i}=\max _{j} d_{i}^{(j)}$, for $0 \leq i \leq p$. Then $l \leq d^{(j)} \leq u$ for $1 \leq j \leq n$. Suppose $l^{\prime} \leq d^{(j)}$ for $1 \leq j \leq n$. Then $l^{\prime}{ }_{i} \leq d_{i}^{(j)}$ for $0 \leq i \leq p$ and $1 \leq j \leq n$, so $l^{\prime} \leq l$ and $l$ is the meet of $D$. Similarly, $u$ is the join of $D$.

Notice further that, by definition, $\underline{d} \leq d \leq \bar{d}$ for all $d \in \Pi_{\underline{d}, \bar{d}}$. Thus, $\Pi_{\underline{d}, \bar{d}}$ is a bounded complete lattice. 
Next we see that reversing the order on $\Pi_{d, \bar{d}}$ yields a (possibly different) BoijSöderberg poset.

Proposition 2.2. Let $\widetilde{\Pi_{\underline{d}}, \bar{d}}$ be the set $\Pi_{\underline{d}, \bar{d}}$ endowed with the partial ordering given by $d \leq_{\sim}$ e if $d_{i} \geq e_{i}$ for $0 \leq i \leq p$. Then $\widetilde{\Pi_{\underline{d}, \bar{d}}}$ is isomorphic to some Boij-Söderberg poset.

Proof. Without loss of generality, assume $\underline{d}_{0}=0$. Let $m=\bar{d}_{p}$ and define the map $\varphi$ from $\{0, \ldots, m\}$ onto itself by $i \mapsto m-i$ and extend $\varphi$ componentwise to $\{0, \ldots, m\}^{p+1}$. Further, define the map $\rho$ from $\{0, \ldots, m\}^{p+1}$ onto itself by $\left(d_{0}, \ldots, d_{p}\right) \mapsto\left(d_{p}, d_{p-1}, \ldots, d_{0}\right)$. Notice $\rho \circ \varphi$ is a bijection.

Then for $d, e \in \Pi_{\underline{d}, \bar{d}}$, we have that

$$
\begin{aligned}
d \leq e & \Leftrightarrow d_{i} \leq e_{i}, 0 \leq p \\
& \Leftrightarrow \varphi\left(d_{i}\right)=m-d_{i} \geq \varphi\left(e_{i}\right)=m-e_{i}, 0 \leq p \\
& \Leftrightarrow \rho(\varphi(d)) \geq \rho(\varphi(e)) .
\end{aligned}
$$

That is, $\rho \circ \varphi$ exactly reverses the order of elements in $\Pi_{\underline{d}, \bar{d}}$, and hence $\Pi_{\rho(\varphi(\bar{d})), \rho(\varphi(\underline{d}))}$ is isomorphic to $\widetilde{\Pi_{\underline{d}, \bar{d}}}$.

An immediate result of this is that particular posets are isomorphic to themselves after reversing the order.

Corollary 2.3. Let $b, m \in \mathbb{N}$ and suppose $\underline{d}=(0, m, 2 m, \ldots, p m)$ and $\bar{d}=(b, m+$ $b, 2 m+b, \ldots, p m+b)$. Then $\Pi_{\underline{d}, \bar{d}}$ is isomorphic to $\widetilde{\Pi_{\underline{d}}, \bar{d}}$.

Proof. Let $\varphi$ and $\rho$ be as in the proof of Proposition 2.2 recall that $\rho \circ \varphi$ is a bijection. Applying $\rho \circ \varphi$ to $\underline{d}$, we get

$$
\begin{aligned}
\rho(\varphi(\underline{d})) & =(p m+b-p m, p m+b-(p-1) m, \ldots, p m+b-m, p m+b-0) \\
& =(b, m+b, \ldots,(p-1) m+b, p m+b) \\
& =\bar{d} .
\end{aligned}
$$

Thus, $\rho \circ \varphi$ is a poset isomorphism; that is, $\Pi_{\rho(\varphi(\bar{d})), \rho(\varphi(\underline{d}))} \cong \Pi_{\underline{d}, \bar{d}}$.

This then allows us to see that some posets can be simplified.

Proposition 2.4. Suppose $\underline{d}=(0, \ldots, p)$ and $\bar{d}=(k, \ldots, p+k)$ for some $1 \leq k \leq p$. Then

$$
\Pi_{\underline{d}, \bar{d}} \cong \Pi_{(0, \ldots, k-1),(p+1, \ldots, p+k)} .
$$

Proof. By Corollary $2.3, \Pi_{\underline{d}, \bar{d}} \cong \widetilde{\Pi_{\underline{d}, \bar{d}}}$. Let $g:\left(\begin{array}{c}\{0, \ldots, p+k\} \\ p+1\end{array}\right) \rightarrow\left(\begin{array}{c}\{0, \ldots, p+k\} \\ k\end{array}\right)$ be given by $A \mapsto\{0, \ldots, p+k\}-A$.

Extending $g$ componentwise provides a bijective map from elements of $\widetilde{\Pi_{d}, \bar{d}}$ to elements of $\Pi_{(0, \ldots, k-1),(p+1, \ldots, p+k)}$. Further still, for $d, e \in \widetilde{\Pi_{d, \bar{d}}}$, we have that $d \leq_{\sim} e$ if and only if the $i^{\text {th }}$ largest missing element of $e$ is at least the $i^{\text {th }}$ largest missing element of $d$ for all $1 \leq i \leq k$. That is, $d \leq_{\sim} e$ if and only if $g(d) \leq g(e)$. Hence $g$ is a poset isomorphism.

The binomial coefficients and the multi-dimensional Catalan numbers give the number of vertices and the number of facets, respectively, for Boij-Söderberg posets with upper and lower sequences given by consecutive integers. 
Lemma 2.5. Let $\underline{d}=(0, \ldots, p)$ and $\bar{d}=(k, \ldots, p+k)$ for some positive integer $k$. Then $\Pi_{\underline{d}, \bar{d}}$ has $\left(\begin{array}{c}p+\bar{k}+1 \\ p+1\end{array}\right)$ vertices and

$$
f(p, k):=(p k+k) ! \prod_{i=0}^{p} \frac{i !}{(k+i) !}
$$

facets.

Proof. Every vertex of $\Pi_{d, \bar{d}}$ is a sequence of $p+1$ numbers which can be seen as a $p+1$ subset of $\{1,2, \ldots, p+k\}$. Similarly, every such $p+1$ subset can be seen as a strictly increasing sequence of $p+1$ numbers. Hence there are $\left(\begin{array}{c}p+k+1 \\ p+1\end{array}\right)$ vertices.

Consider the vertices of $\Pi_{d, \bar{d}}$ with $\underline{d}$ subtracted from them. Then we are considering weakly increasing sequences of length $p+1$ with entries from $\{0,1, \ldots, k\}$. Thus under the aforementioned consideration of the vertices, the maximal chains in $\Pi_{d, \bar{d}}$ then correspond directly to the $\mathrm{SU}(p+1)$ walk diagrams of $(p+1) k$-steps as described in [6, Section IV]. Moreover, the number of such walk diagrams is given in [6, Equation (4.8)] as $f(p, k)$.

As every Boij-Söderberg poset contains and is contained in such a poset, we can give a bound on the number of vertices and number of facets of an arbitrary Boij-Söderberg poset.

Corollary 2.6. Let $\underline{d}, \bar{d} \in \mathbb{Z}_{0}^{p+1}$ be strictly increasing sequences with $\underline{d}_{i} \leq \bar{d}_{i}$ for $0 \leq i \leq p$. If we set $v$ to be the number of vertices and $n$ to be the number of facets of $\Pi_{\underline{d}, \bar{d}}$, then

$$
\left(\begin{array}{c}
\bar{d}_{0}-\underline{d}_{p}+2 p+1 \\
p+1
\end{array}\right) \leq v \leq\left(\begin{array}{c}
\bar{d}_{p}-\underline{d}_{0}+1 \\
p
\end{array}\right)
$$

and

$$
f\left(p, \bar{d}_{0}-\underline{d}_{p}+p\right) \leq n \leq f\left(p, \bar{d}_{p}-\underline{d}_{0}-p\right) .
$$

We further make the observation that vertex-decomposability of $\Delta\left(\Pi_{\underline{d}}, \bar{d}\right)$ implies vertex-decomposability of any $\Delta\left(\Pi_{\underline{d}^{\prime}, \bar{d}^{\prime}}\right)$ where $\underline{d} \leq \underline{d}^{\prime} \leq \bar{d}^{\prime} \leq \bar{d}$.

Remark 2.7. Let $d \in \Pi_{\underline{d}, \bar{d}}$. Then $\operatorname{link}_{\Delta\left(\Pi_{\underline{d}, \bar{d}}\right)} d=\Gamma_{d} \star \Sigma_{d}$, where $\Gamma_{d}=\Delta(\{e \in$ $\left.\left.\Pi_{\underline{d}, \bar{d}} \mid e<d\right\}\right)$ and $\Sigma_{d} \stackrel{\underline{=}}{=} \Delta\left(\left\{e \in \Pi_{\underline{d}, \bar{d}} \mid \underline{d}<e\right\}\right)$. Hence $d \star \Gamma_{d}=\Delta\left(\Pi_{\underline{d}, d}\right)$ and $d \star \Sigma_{d}=\Delta\left(\Pi_{d, \bar{d}}\right)$.

For $\underline{d} \leq \underline{d}^{\prime} \leq \bar{d}^{\prime} \leq \bar{d}$, we then have that $\Delta\left(\Pi_{\underline{d}^{\prime}, \bar{d}^{\prime}}\right)$ is obtained from $\Delta\left(\Pi_{\underline{d}, \bar{d}}\right)$ by linking, taking half of a join, and coning. Thus, properties like vertex-decomposability and Cohen-Macaulayness, which respect linking, joining, and coning, are preserved.

\section{Recursive Structure}

Boij-Söderberg posets admit recursive atom orderings which are simple to describe.

Theorem 3.1. Let $\underline{d}, \bar{d} \in \mathbb{Z}_{0}^{p+1}$ be strictly increasing sequences with $\underline{d}_{i} \leq \bar{d}_{i}$ for $0 \leq i \leq p$. Then $\Pi_{\underline{d}, \bar{d}}$ admits a recursive atom ordering when the atoms are ordered lexicographically from smallest to largest. 
Proof. Let $\underline{d}, \bar{d} \in \mathbb{Z}_{0}^{p+1}$ be strictly increasing sequences with $\underline{d}_{i} \leq \bar{d}_{i}$ for $0 \leq i \leq p$; assume without loss of generality that $\underline{d}_{i}<\bar{d}_{i}$ for $0 \leq i \leq p$ (see [4, Lemma 3.3]).

If $\underline{d}$ and $\bar{d}$ differ in only one position, then $p=0$ and clearly $\Pi_{\underline{d}, \bar{d}}$ admits a recursive atom ordering. Suppose then that $\underline{d}$ and $\bar{d}$ differ in more than one position, i.e., $p>0$. Define $e_{i} \in \mathbb{Z}^{p+1}$ by $\left(e_{i}\right)_{j}=0$ if $j \neq i$ and $\left(e_{i}\right)_{i}=1$.

The atoms of $\Pi_{d, \bar{d}}$ are exactly the elements $\underline{d}+e_{i}$ where $\underline{d}_{i}+1 \leq \underline{d}_{i+1}$ or $i=p$; let $i_{1}>\cdots>i_{t}$ be the indices of the atoms. Then under the lexicographic ordering, $\underline{d}+e_{i_{1}}<\cdots<\underline{d}+e_{i_{m}}$. Furthermore, by induction on the number of positions where $\underline{d}$ and $\bar{d}$ differ, we may assume that $P_{j}=\Pi_{\underline{d}+e_{i_{j}}, \bar{d}}$ admits a recursive atom ordering when the atoms are ordered lexicographically from smallest to largest.

For $j \geq 2$, the atom $\underline{d}+e_{i_{j}}+e_{i_{k}}$ of $P_{j}$ covers $\underline{d}+e_{i_{k}}$ for $1 \leq k<j$, and these are the only atoms of $P_{j}$ which cover some $\underline{d}+e_{i_{k}}$ for $1 \leq k<j$. Moreover, for $\ell>i_{j}$ not equal to some $i_{k}$ for $1 \leq k<j, \underline{d}_{\ell}+1=\underline{d}_{\ell+1}$, so $\underline{d}+e_{i_{j}}+e_{\ell}$ is not a member of $P_{j}$. Hence, the atoms $\underline{d}+e_{i_{j}}+e_{i_{k}}$, for $1 \leq k<j$, of $P_{j}$ are those which come first in the ordering of $P_{j}$.

For $1 \leq k<j \leq t$, the atom $z=\underline{d}+e_{i_{j}}+e_{i_{k}}$ of $P_{j}$ is the join of $\underline{d}+e_{i_{j}}$ and $\underline{d}+e_{i_{k}}$ and covers both atoms. Hence if $\underline{d}+e_{i_{k}}, \underline{d}+e_{i_{j}}<y$ for some $y \in \Pi_{d, \bar{d}}$, then $z \leq y$.

Therefore $\Pi_{\underline{d}, \bar{d}}$ admits a recursive atom ordering when the atoms are ordered lexicographically from smallest to largest.

As the poset admits a recursive atom ordering, the associated order complex is vertex-decomposable, hence squarefree glicci (so, in particular, in the Gorenstein liaison class of a complete intersection; see [9]) and Cohen-Macaulay.

Corollary 3.2. All Boij-Söderberg order complexes are vertex-decomposable, hence squarefree glicci and Cohen-Macaulay.

Proof. Let $\underline{d}, \bar{d} \in \mathbb{Z}_{0}^{p+1}$ be strictly increasing sequences with $\underline{d}_{i} \leq \bar{d}_{i}$ for $0 \leq i \leq p$. Then by Theorem [3.1, $\Pi_{\underline{d}, \bar{d}}$ admits a recursive atom ordering. Thus by [1, Theorem 3.2] and [2, Theorem 11.6], the order complex of $\Pi_{d, \bar{d}}$ is vertex-decomposable.

Pure vertex-decomposable simplicial complexes are squarefree glicci 9, Theorem 3.3] and pure shellable [10, Theorem 2.8], hence Cohen-Macaulay.

However, not every Boij-Söderberg order complex remains in the family during shedding.

Remark 3.3. Consider $\underline{d}=(1,3,4)$ and $\bar{d}=(2,5,6)$, which are illustrated in Figure 2. Then the shedding vertices of $\Pi_{d, \bar{d}}$ are $(1,3,6),(1,4,5),(1,5,6),(2,3,4)$, $(2,3,6)$, and $(2,4,5)$.

Removing either $(2,3,4)$ or $(1,5,6)$ creates a situation where a single change is followed by a triplet of changes or the opposite-either case is impossible in our family. Lastly, removing the other four vertices creates a situation which is, after tedious calculation, demonstrably impossible. In particular, the form implies $3 \leq$ $\# d \leq 5$ but also that $\hat{0}$ would have exactly one consecutive pair of entries with nonconsecutive values. Checking the nine possible situations yields a contradiction in each case.

Hence, deleting any shedding vertex from $\Pi_{\underline{d}, \bar{d}}$ yields a new poset which is not a Boij-Söderberg poset. 


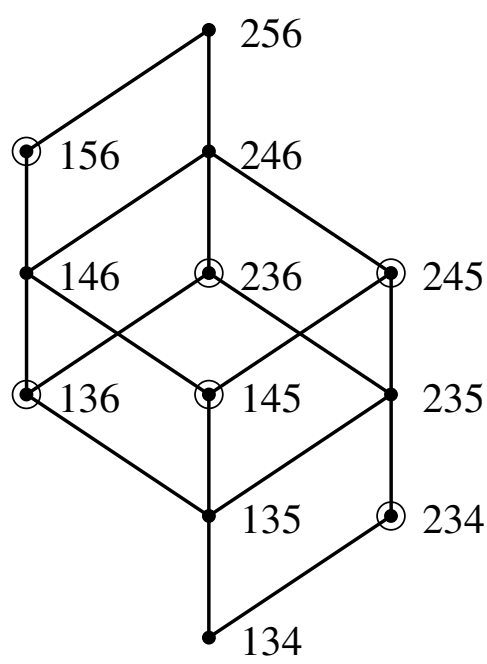

FiguRE 2. The Hasse diagram of $\Pi_{(1,3,4),(2,5,6)}$ with shedding vertices circled

\section{ACKNOWLEDGEMENTS}

The author would like to thank his advisor, Uwe Nagel, for reading drafts of this article and for making comments thereon. The author would also like to thank Heide Gluesing-Luerssen for assistance in the aesthetics of the figures.

\section{REFERENCES}

1. Anders Björner and Michelle Wachs, On lexicographically shellable posets, Trans. Amer. Math. Soc. 277 (1983), no. 1, 323-341. MR690055 (84f:06004)

2. Anders Björner and Michelle L. Wachs, Shellable nonpure complexes and posets. II, Trans. Amer. Math. Soc. 349 (1997), no. 10, 3945-3975. MR.1401765 (98b:06008)

3. Mats Boij and Jonas Söderberg, Betti numbers of graded modules and the multiplicity conjecture in the non-Cohen-Macaulay case, arXiv e-prints (2008).

4. Mats Boij and Jonas Söderberg, Graded Betti numbers of Cohen-Macaulay modules and the multiplicity conjecture, J. Lond. Math. Soc. (2) 78 (2008), no. 1, 85-106. MR 2427053 (2009g:13018)

5. David Cook II, Simplicial decomposability, The Journal of Software for Algebra and Geometry 2 (2010), 20-23.

6. P. Di Francesco, SU(N) meander determinants, J. Math. Phys. 38 (1997), no. 11, 5905-5943. MR:1480837 (99k:05019)

7. David Eisenbud and Frank-Olaf Schreyer, Betti numbers of graded modules and cohomology of vector bundles, J. Amer. Math. Soc. 22 (2009), no. 3, 859-888. MR2505303

8. Daniel R. Grayson and Michael E. Stillman, Macaulay2, a software system for research in algebraic geometry, available at http://www.math.uiuc.edu/Macaulay2/.

9. Uwe Nagel and Tim Römer, Glicci simplicial complexes, J. Pure Appl. Algebra 212 (2008), no. 10, 2250-2258. MR2426505 (2009c:13025)

10. J. Scott Provan and Louis J. Billera, Decompositions of simplicial complexes related to diameters of convex polyhedra, Math. Oper. Res. 5 (1980), no. 4, 576-594. MR.593648(82c:52010)

Department of Mathematics, University of Kentucky, 715 Patterson Office Tower, Lexington, Kentucky 40506-0027

E-mail address: dcook@ms.uky.edu 